\title{
Stabilization of Supported Liquid Membranes by Gelation with PVC
}

\author{
A. J. B. KEMPERMAN, B. DAMINK, TH. VAN DEN BOOMGAARD, H. STRATHMANN \\ Membrane Technology Group, Department of Chemical Technology, University of Twente, P.O. Box 217, \\ NL-7500 AE Enschede, The Netherlands
}

Received 4 September 1996; accepted 20 December 1996

\begin{abstract}
This article describes the gelation by PVC of supported liquid membranes ( SLMs) for nitrate removal using a quaternary ammonium salt as carrier. Untreated SLMs with this carrier are very unstable. To improve their stability, the LM-phase was gelled with polyvinyl chloride as gel-forming polymer. Both homogeneous gelations of the LM-phase as well as the application of an interfacial gel layer are described. In all cases, no improvement of the stability could be observed. For both types of gelation, the initial nitrate flux decreased while the flux after 1 day of degradation was almost zero. The flux decrease is the result of a decrease of the the diffusion rate of carrier complex as a result of the presence of the gel network and the thickness of the applied gel layer. The absence of any stability improvement might indicate that the loss of LMphase from these membranes is due to dissolution of carrier or membrane solvent and not a result of emulsion formation only. (C) 1997 John Wiley \& Sons, Inc. J Appl Polym Sci 65: $1205-1216,1997$
\end{abstract}

Key words: gelation; polyvinylchloride; nitrate removal; facilitated transport; liquid membrane stability

\section{INTRODUCTION}

Supported liquid membranes (SLMs), in which an organic solvent containing a carrier is immobilized in the pores of a microfiltration membrane, is a promising membrane separation technology. However, their lack of stability, and subsequently short lifetime, is the main reason these membranes are not applied at a large industrial scale. One of the possible reasons for the instability of SLMs, besides the solubility of the organic solvent and/or the carrier in the aqueous feed and stripping phases, might be an emulsification of the LM-phase induced by lateral shear forces. ${ }^{1-5}$

The stability of SLMs can be improved by gelation of the liquid membrane phase, as has been

\footnotetext{
Correspondence to: Thonie van den Boomgaard (A. vandenBoomgaard@ct.utwente.nl).

(C) 1997 John Wiley \& Sons, Inc. CCC 0021-8995/97/061205-12
}

shown by Neplenbroek et al. ${ }^{1,6,7}$ According to Tanaka, a gel consists of polymers or long-chain molecules crosslinked to create a tangled network and immersed medium. The polymer network is prevented from collapsing into a compact mass by the liquid, while the liquid is prevented from flowing away by the network. ${ }^{8}$

From the definition of a polymer gel given above, it might be clear that the nature of the liquid itself is not changed, but there are polymer chains ("obstacles") present in the liquid. For carrier-mediated transport membranes like SLMs, this enables one to make use of gelation for embedding the solvent in a polymer matrix while the carrier molecules, necessary for transport, are still dissolved in the liquid. The only drawback will be the slower kinetics of transport inside the carrier-mediated transport membrane. This will depend on the number of obstacles (the polymer concentration in the gel) and the crosslink density. 
The principle of gelation has been used by other researchers for different membrane applications. Bloch et al. were the first who used so-called "solvent membranes" for the selective transport of metal ions. ${ }^{9}$ These solvent membranes were made of polyvinyl chloride (PVC), plasticized with phosphate esters like tributylphosphate (TBP), cresyldibutylphosphate (CDBP), or dicresylbutylphosphate (DCBP). The phosphates could act as an extracting agent for the separation of uranium from aqueous solutions. However, the lifetime of these membranes was insufficient as a result of loss of plasticizer, resulting in completely impermeable, hard PVC films. Vofsi et al. extended research on solvent membranes to other systems and to the use of this type of membrane as ionselective electrodes. ${ }^{10-12}$ The combination of polymer films and plasticizers was also investigated by Sugiara et al. for the transport of picrate or metal ions. ${ }^{13-16}$ Due to the thickness and high polymer concentrations of these "solvent polymeric membranes" necessary for a sufficient mechanical strength, fluxes were up to a factor 100 lower than described throughout this article. Gankema prepared solvent polymeric membranes by gel casting of solutions of ultra-high molecular weight polyethylene (UHMW-PE) and a carrier solution. ${ }^{17}$ It was claimed that the stability of these systems could be improved by changing the drawing ratio. Sakahora et al. prepared acrylamide gels in the pores of silica-alumina membranes for the pervaporation of organic/water mixtures. ${ }^{18}$ Membrane performance did not change significantly for a period of three months. In a patent by Matson et al., the application of a thin gel layer on top of a microporous polymeric support was reported for the scrubbing of acid gases. ${ }^{19}$

The idea of improving the stability of SLMs by a homogeneous gelation in the pores of the support was first suggested by Neplenbroek et al. ${ }^{1,6,7}$ The support improved the mechanical strength of the gel, while the presence of the gel could prevent loss of the membrane phase in emulsion droplets, as was expected by the authors. The gel would effectively prevent the deformation of the liquid membrane (LM) meniscus, as is shown schematically in Figure 1. In turn, this would lead to a much more difficult formation of emulsions of the LM-phase in the aqueous phases, and thus to a higher stability of the SLM.

Indeed, promising results were obtained using PVC as polymer and tetraoctylammonium bromide ( $\mathrm{TeOA}-\mathrm{Br}$ ) (which already gave fairly stable

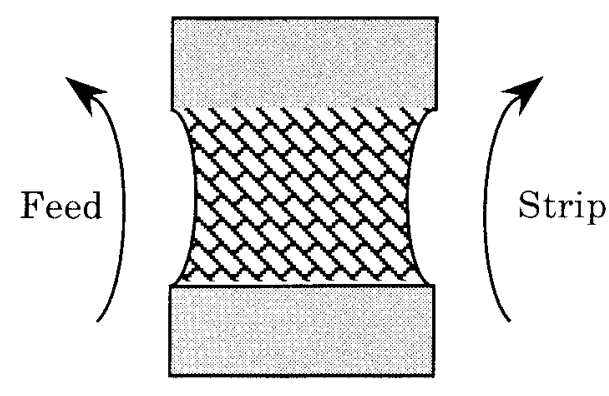

(a)

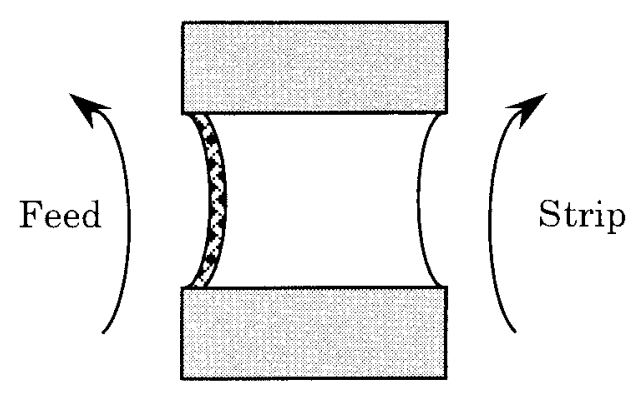

(b)

Figure 1 Schematic drawing illustrating the stabilization of SLMs by gelation. (a) Homogeneous gel network in the support pores; (b) Thin dense gel layer at feed side.

SLMs without gelation) as carrier. The motivations for the use of this polymer are the following. The material is hydrophobic, and the LM-phase can be used as solvent for the gel-forming polymer since $o$-nitrophenyloctylether (o-NPOE), the membrane solvent, is known to be a plasticizer for PVC. ${ }^{20}$ It is possible to obtain a gelled phase already at low polymer concentrations $(2-3$ wt \% for PVC/di-2-ethylhexylphthalate ).$^{21}$ Furthermore, in related systems like ion-selective electrodes, this polymer is most frequently applied ${ }^{20}$ and superior in stability and sensitivity to polyurethane, ${ }^{22,23}$ polymethylmethacrylate, polystyrene, and silicone rubber ${ }^{22}$ membranes. However, the best results were obtained when the gel was applied as a thin layer $(<2 \mu \mathrm{m})$ on the feed side of the SLM [Fig. 1(b)] and chemically crosslinked after preparation. In this way, the resistance toward transport as a result of gelation was minimized as a result of the low thickness of the gel layer. Secondly, emulsion formation of the LMphase, according to the authors the main reason of SLM instability in their system, could be suppressed more effectively since the polymer concen- 
tration could be high without any flux reduction. Two-month experiments were carried out under circumstances that significantly enhanced the degradation of the SLM. A membrane lifetime over two years under normal circumstances was predicted by the authors. Other research on gelled SLMs, based on Neplenbroek's promising results, was carried out by Bromberg, Levin, and Kedem ${ }^{24}$ and Levin and Bromberg. ${ }^{25}$ Both authors also used PVC as gel-forming polymer and are working on changing the carrier. However, no data on membrane lifetime were presented.

Neplenbroek et al. only presented a few data on long-term stability of gelled membranes using very unstable SLMs with trioctylmethylammonium chloride (TOMA-Cl) as carrier. Therefore, in this article the preparation and characterization of homogeneously gelled SLMs and SLMs with a gelled top layer using this carrier are described using PVC as gel-forming polymer.

\section{EXPERIMENTAL}

\section{Materials and Membranes}

\section{Support}

Throughout this article, two different flat sheet supports were used. Accurel ${ }^{\circledR} 1 \mathrm{E}-\mathrm{PP}$ was obtained from Akzo. It is made from polypropylene, has a nominal pore diameter of $0.2 \mu \mathrm{m}$, an overall porosity of $69 \%$ (Akzo specifications), and a thickness of $\sim 100 \mu \mathrm{m}$ (measured with a Mitutoyo digital micrometer ). Durapore GVHP (Millipore) is a hydrophobic PVDF membrane with a pore size of $0.22 \mu \mathrm{m}$, a thickness of $80 \mu \mathrm{m}$, and $75 \%$ overall porosity (data from Millipore).

\section{Carrier and Solvent}

In all cases, o-nitrophenyloctylether (o-NPOE) was used as membrane solvent for the quaternary ammonium salts used as carriers. The $o$-NPOE was synthesized in our laboratories by a reaction of $o$-nitrophenol and bromooctane. ${ }^{26}$ As carrier, both TOMA-Cl and TeOA-Br were applied. The carriers were obtained from Fluka and used without further purification. Carrier concentration in the LM-phase was always $0.2 \mathrm{M}$. The TOMA/oNPOE mixture was heated slightly to dissolve all the carrier in a fast way, after which the solution remained clear at room temperature.

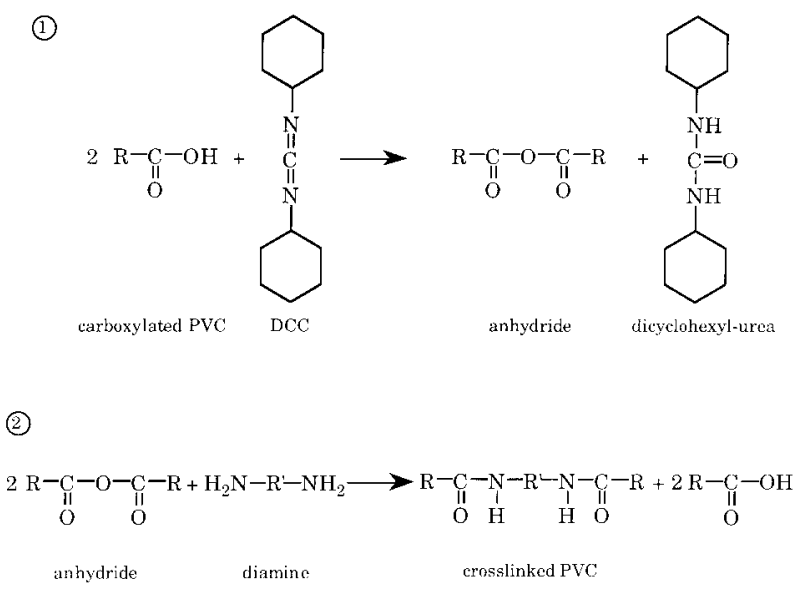

Figure 2 Chemical crosslinking of carboxylated PVC. (1) The PVC is activated by dicyclohexylcarbodiimide (DCC); (2) Two coupled PVC chains react with a diamine. Adapted from Neplenbroek et al. ${ }^{1,3}$

\section{Gelation}

PVC was used for the gelation of the LM-phase. PVC-HMW ("high molecular weight") and PVCLMW ("low molecular weight") were obtained from Fluka. The molecular weight of PVC-LMW was 48,000 (supplier's data), while the molecular weight value for PVC-HMW as determined by gel permeation chromatography was $91,000 .{ }^{1,7}$ Tetrahydrofuran (THF, Merck) was used for gel preparation by evaporation of a volatile second solvent. After complete evaporation of THF, a reversible, physically crosslinked PVC gel results; the PVC content is too low to obtain a plasticized polymer film. Chemically crosslinked gels were obtained by using carboxylated PVC (PVC-carb) from Janssen Chimica. Its molecular weight was 200,000, with $1.8 \mathrm{wt} \%$ carboxyl groups. As activator for the chemical crosslinking reaction, $N, N^{\prime}$-dicyclohexylcarbodiimide (DCC) from Janssen Chimica was used. 1,4-Phenylenediamine, PDA, (Fluka) or 1,12-diaminododecane, DDDA, (Janssen Chimica) were chosen as crosslinking agents. The crosslinking reaction is shown schematically in Figure 2. The chemical crosslinking reaction was carried out to obtain a gel with irreversible crosslinks, which might show a more constant behavior in time.

All these chemicals were used as received without further purification. PVC-HMW, the activator DCC and both crosslinkers had the same product specifications as those used by Neplenbroek et al. ${ }^{1,7}$ The PVC-carb used in this article contained a slightly higher amount of carboxyl groups than that applied by them. 


\section{Membrane Preparation}

\section{Homogeneous Gelation}

Homogeneously gelled SLMs were prepared in two different ways, by the volatile solvent method and by a temperature method. For the volatile solvent method, the desired amount of PVC was dissolved in the LM-phase (0.2 $M$ TOMA-Cl in $o$ NPOE). To this mixture, a certain amount of THF was added ( see Table I). The amount of THF used depended on the polymer concentration. The higher the PVC content, the higher the viscosity of the PVC/LM-phase/THF mixture and the less easily the support got impregnated with the gelation mixture. Therefore, the amount of THF used was higher when the PVC content was higher. The THF, polymer, and LM-phase were stirred together until a homogeneous mixture was formed. This solution was poured out in a petri dish, after which the LM-support (74-mm diam.) was placed in the solution. After at least one day of drying under nitrogen, all THF was assumed to be evaporated entirely since no further mass decrease could be observed. Residual gel substance was carefully removed from the surface of the membrane by gently wiping with a paper tissue. In all these cases, PVC-HMW was used as polymer.

When the temperature method was used for the gel preparation, the desired amounts of PVC and LM-phase were put in a petri dish. The dish was placed in an oven $\left(120^{\circ} \mathrm{C}\right)$ under nitrogen for half an hour and stirred from time to time, after which the support (74-mm diam.) was placed in the homogeneous mixture. After $3 \mathrm{~min}$, the impregnated support was removed from the dish and remaining gel substance at the surface was wiped off quickly by means of a tissue. The different actions outside the oven had to be carried out quickly, since immediately after removing the membrane from the oven gel formation started, and as a result the remaining gel could not be removed.

Table I Amount of THF Added to the Different Amounts of PVC/LM-Phase Mixtures for the Preparation of Homogeneously Gelled SLMs

\begin{tabular}{cc}
\hline PVC (wt \%) & THF/g Mixture (mL) \\
\hline 2.5 & 5.0 \\
5.0 & 7.5 \\
7.5 & 10.0 \\
10.0 & 12.5 \\
\hline
\end{tabular}

Table II Amount of THF Added to the Different Amounts of PVC/LM-Phase Mixture for the Preparation of Thin Gel Layers

\begin{tabular}{cc}
\hline PVC (wt \%) & THF/g Mixture (mL) \\
\hline 40 & 5.0 \\
50 & 6.0 \\
70 & 6.0 \\
100 & 7.0 \\
\hline
\end{tabular}

\section{Interfacial Gel Layers}

A rectangular piece of support (dimensions $\sim 14$ $\times 23 \mathrm{~cm}$ ) was impregnated with the LM-phase. Excess LM-phase was removed using a paper tissue. PVC/LM-phase mixtures were prepared using the desired weight fractions. Different types of PVC were used in this case. Depending on the quantity of polymer in the mixture, a certain amount of THF was added to this mixture (see Table II) and stirred until the solution was homogeneous. To this solution activator and crosslinker, if used, could be added shortly before preparation of the gel layer. Directly after addition of crosslinker and/or activator, the homogeneous solution was spread on the impregnated support, which was placed on a horizontal glass plate. By means of casting knives of $50 \mu \mathrm{m}$ or $100 \mu \mathrm{m}$, the solution was spread as a thin layer of uniform thickness on top of the impregnated support. The membranes were stored under nitrogen for at least three days before use. The thickness of the gel layers was measured with a digital Mitutoyo micrometer. By means of this technique, developed in our laboratories by Wijers, ${ }^{27}$ the layers were much more uniform in thickness than those prepared by the method of Neplenbroek et al. using a tissue. ${ }^{1,7}$

\section{Permeability Measurements}

\section{SLM Preparation}

Supported liquid membranes that were not gelled were prepared by spreading LM-phase on the bottom of a petri dish, in which a support (74-mm diam.) was placed. After an impregnation time of at least $15 \mathrm{~min}$, the supports were completely transparent and were removed from the dish. Attached membrane phase was carefully wiped from the surface of the SLM. The preparation of homogeneously gelled SLMs was described before. From the sheet SLM with an interfacial gel layer, 
a piece (74-mm diam.) was carefully cut out using a scalpel. In some cases, SLMs with a gel layer on both sides of the membrane were used. They were prepared using two SLMs with one gel layer each, which were "glued" together using a droplet of LM-phase.

\section{Flux Determination}

In all cases, the flux was determined in the same way as described before by Neplenbroek et al. ${ }^{1,28}$ in a setup where feed and strip solutions were flowing parallel to the SLM interface. The gel layer was directed toward the feed side. The feed consisted of $130 \mathrm{~mL} 4 \times 10^{-3} \mathrm{M} \mathrm{NaNO}_{3}$ (Merck) solution in water; the strip phase consisted of 130 $\mathrm{mL} 4 M \mathrm{NaCl}$ (Merck). Both phases were pumped around with a velocity of $5.5 \mathrm{~mL} \mathrm{~s}^{-1}$ using Masterflex ${ }^{\circledR}$ peristaltic pumps. By observing the concentration of nitrate and chloride in the feed phase in time (HPLC, Waters IC-PAK ${ }^{\mathrm{TM}}$ anion column), the nitrate flux was calculated.

\section{Stability Measurements}

In between two flux measurements, the feed phase was replaced by a $10^{-4} M \mathrm{NaNO}_{3}$ solution. A lower salt concentration enhances degradation of the membrane as was observed by Neplenbroek et al. ${ }^{1,3}$ After $\sim 20-21 \mathrm{~h}$ of "degradation," both feed and strip were refreshed with the original solutions for a new determination of the nitrate flux. In this way an uncoated membrane, with TOMA-Cl as carrier, showed no nitrate transport after 1 day of degradation. In several cases, longterm experiments were carried out over a period up to 7 days. The masses of the SLMs before and after the flux measurements were determined using an analytical balance to obtain the LM-phase loss.

\section{Characterization}

SLMs with an interfacial gel layer were examined by scanning electron microscopy (SEM) to investigate the uniformity of the gel layer thickness and the texture of the gel layer. Cross sections were prepared by breaking small sections of the gelled membrane in liquid nitrogen, after which they were placed in a sample holder. Surface samples were simply cut out of the membrane and glued on a sample holder by means of double-sided adhesive tape. The samples were sputtered with gold using a Balzer Union SCD 040 sputtering apparatus (sputtering time $3 \mathrm{~min}$ at $15 \mathrm{~mA}$ ). SEM investigations were carried out on a Jeol JSM T 220A scanning electron microscope at 20 $\mathrm{kV}$. Despite the presence of gelled LM-phase, no problems were met in obtaining the high vacuum necessary in the apparatus.

\section{RESULTS}

\section{Homogeneous Gelation}

\section{Temperature Method}

Serious problems were met when homogeneously gelled SLMs were prepared by the temperature method at $120^{\circ} \mathrm{C}$. A color change of the PVC-LMphase mixture was observed 10-15 min after the mixture was placed in the oven. The color changed from yellow via red to dark, almost black. The color change was not observed in absence of polymer. This might indicate a degradation of the PVC since it is known that certain PVC polymers are easily thermally and photochemically degraded. ${ }^{29}$ To investigate whether the degradation was a result of the high temperature, the presence of carrier and organic solvent, or a combination of these possibilities, several experiments were carried out. In an oven, small amounts of PVC, PVC $+o-$ NPOE, PVC + TOMA-Cl or TeOA-Br, o-NPOE, and carrier were placed and heated under nitrogen at $120^{\circ} \mathrm{C}$ for half an hour. Only the $\mathrm{PVC} /$ carrier mixtures showed a change in color. This is an indication that the presence of carrier enhances degradation of the PVC. Neplenbroek et al. also encountered these PVC-degradation problems with PVC from Fluka, but no degradation problems were met when PVC from BASF was used. ${ }^{1,7}$ This might be explained by the presence of stabilizers in the PVC-BASF, which are usually added to commercial PVC in order to minimize degradation of the polymer.

Despite the PVC degradation problems, these homogeneously gelled SLMs were tested on nitrate flux and stability. The results are given in Table III. The 0 wt \% PVC membranes were prepared in the oven to investigate the effect of the heat treatment on nitrate flux and SLM stability. No difference is found with values for reference membranes not treated in an oven. Addition of PVC to the membrane phase results in a small decrease of the initial nitrate flux since the microscopic viscosity influencing the diffusion rate of the carrier-ion complex remains nearly constant. Since SLMs prepared with the volatile solvent 
Table III Nitrate Fluxes and Stability of Homogeneously Gelled PVC SLMs

\begin{tabular}{ccccc}
\hline $\begin{array}{c}\text { PVC Content } \\
(\text { wt \%) }\end{array}$ & PVC Type & $\begin{array}{c}\text { Initial Flux } \\
\left(10^{-10} \mathrm{~mol} \mathrm{~cm}^{-2} \mathrm{~s}^{-1}\right)\end{array}$ & $\begin{array}{c}\text { Flux After 1 Day } \\
\left(10^{-10} \mathrm{~mol} \mathrm{~cm}^{-2} \mathrm{~s}^{-1}\right)\end{array}$ & $\begin{array}{c}\text { LM Loss } \\
(\text { wt \%) }\end{array}$ \\
\hline 0 & - & 15.6 & 1.7 & 5.6 \\
0 & - & 17.8 & 1.4 & 6.5 \\
2.5 & PVC-HMW & 14.7 & 1.4 & 6.1 \\
2.5 & PVC-HMW & 15.3 & 1.5 & 6.3 \\
2.5 & PVC-LMW & 14.3 & 1.3 & 5.7 \\
2.5 & PVC-LMW & 14.7 & 1.1 & 5.2 \\
\hline
\end{tabular}

Support, Accurel; carrier, TOMA-Cl; gelation at $120^{\circ} \mathrm{C}$.

method showed almost identical initial nitrate fluxes at $2.5 \mathrm{wt} \% \mathrm{PVC}$ ( see next section), we assume that the heat treatment and PVC degradation do not decrease the activity of the carrier. No improvement in SLM stability was observed; in all cases, the flux after 1 day was nearly zero and the LM loss did not differ from that of the reference membranes. The use of different types of PVC did not give differences in nitrate fluxes.

\section{Volatile Solvent Method}

Homogeneously gelled SLMs using THF as volatile third component were prepared as described in the experimental section. It was observed that the use of $5 \mathrm{~mL}$ THF/g PVC/LM-phase mixture as used by Neplenbroek et al. ${ }^{1,7}$ was not satisfactory. The viscosity of the gel was soon too high before the support was fully impregnated. Therefore, more THF was used. However, the attached gel on the surface of the $10 \mathrm{wt} \%$ PVC membranes was far too viscous to be removed using a tissue. Hence, the $10 \mathrm{wt} \%$ PVC membranes were not tested on nitrate transport since difficulties in reproducibility of the results were expected, and research was limited to membranes with a PVC content between 0 and $7.5 \mathrm{wt} \%$.

In Figure 3, the results of the these measurements are plotted. The $0 \mathrm{wt} \% \mathrm{PVC}$ membranes are obtained by adding $5 \mathrm{~mL}$ THF to $1 \mathrm{~g}$ of LMphase and subsequently impregnating the support with the mixture. The flux for an uncoated $0.2 M$ TOMA-Cl SLM is in the order of $16-19$ $\times 10^{-10} \mathrm{~mol} \mathrm{~cm}^{-2} \mathrm{~s}^{-1}$. So, addition of THF to the LM-phase has no influence on the initial nitrate flux. It is clear that addition of PVC to the LMphase results in a decrease of the initial nitrate flux. Despite the increase of the macroscopic viscosity as observed during membrane preparation, the flux decrease is relatively small, indicating that the diffusion rate through the membrane is determined by the microscopic viscosity. The lower fluxes are a result of the fact that the carrier complexes have to travel around the polymer chains in the membrane, resulting in an increase in tortuosity and consequently in a decrease of the flux.

As already revealed by the homogeneously gelled SLMs by the temperature method (Table III), no improvement in stability could be observed for all PVC concentrations investigated. In all cases, the flux after 1 day was nearly zero, while LM losses were all in the order of 14-30 mg. It is expected that when emulsion formation would be the main reason for the instability of TOMA-SLMs, gelation should increase the stability. The opposite is found experimentally.

In Table IV, similar trends can be seen for homogeneously gelled SLMs using Durapore as support. By increasing the polymer content, nitrate flux decreases. The initial fluxes are smaller than those in Figure 3 as a result of differences in support thickness, structure, and porosity. Again, just like the Accurel SLMs, no improvement of the

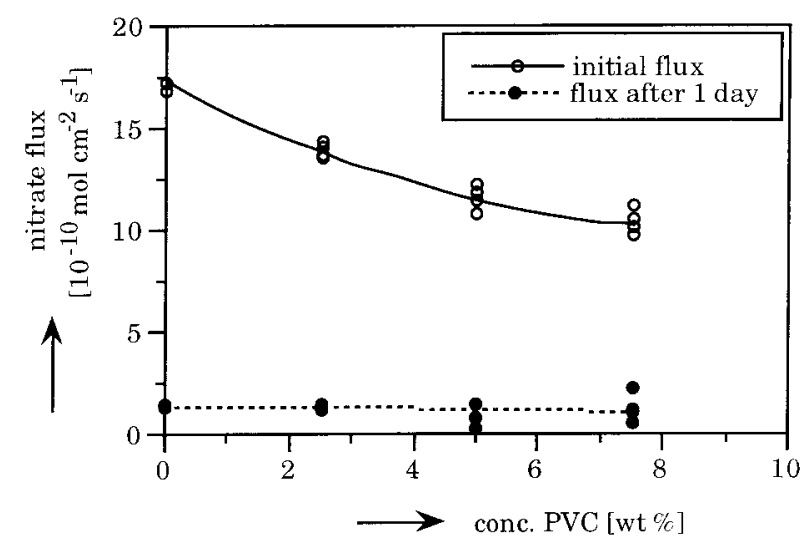

Figure 3 Nitrate fluxes and stability of homogeneously gelled PVC SLMs. Support, Accurel; carrier, TOMA-Cl. Gelation by evaporation of THF. 
Table IV Nitrate Fluxes and Stability of Homogeneously Gelled PVC SLMs

\begin{tabular}{cccc}
\hline $\begin{array}{c}\text { PVC Content } \\
(\text { wt \% })\end{array}$ & $\begin{array}{c}\text { Initial Flux } \\
\left(10^{-10} \mathrm{~mol} \mathrm{~cm}^{-2} \mathrm{~s}^{-1}\right)\end{array}$ & $\begin{array}{c}\text { Flux After 1 Day } \\
\left(10^{-10} \mathrm{~mol} \mathrm{~cm}^{-2} \mathrm{~s}^{-1}\right)\end{array}$ & $\begin{array}{c}\text { LM Loss } \\
(\mathrm{mg})\end{array}$ \\
\hline 0 & 11.0 & 1.1 & 4.8 \\
0 & 11.8 & 1.1 & 5.5 \\
2.5 & 8.3 & 1.1 & 5.9 \\
2.5 & 7.5 & 1.8 & 8.3 \\
7.5 & 6.2 & 0.7 & 5.8 \\
7.5 & 6.2 & 0.5 & 5.1 \\
\hline
\end{tabular}

Support, Durapore 0.22; carrier, TOMA-Cl; gelation by evaporation of THF.

stability was observed. LM losses were in general equal to or a few percent higher than the reference membrane in Table IV.

\section{Gel Layers}

\section{Gel Layers without Chemical Crosslinking}

First, interfacial gel layers were prepared without a chemical crosslinking reaction. The results are given in Table $\mathrm{V}$.

The decrease of the initial nitrate flux with roughly one-third, as a result of the application of the gel layer, is clear in Table V. The volume ratio THF to LM-phase is, for the $40 \%$ membranes, 5 to 1 . This means that the film roughly shrinks to one-sixth of its original thickness during evaporation of THF. Casting with a $100-\mu \mathrm{m}$ knife should therefore give a final gel layer thickness of $\sim 17 \mu \mathrm{m}$. SEM observations showed a somewhat lower thickness $(10 \mu \mathrm{m})$, possibly as a result of a collapse of the gel network in the vacuum of the microscope by evaporation of the or- ganic plasticizer o-NPOE. This layer thickness is higher than that calculated by Neplenbroek et al., who report values of $<2 \mu \mathrm{m}$ obtained with their method. ${ }^{1,7}$ In combination with the high polymer concentration, perhaps our layers are too thick to obtain nitrate fluxes equal to those of uncoated SLMs. The gel network will therefore hinder the transport of the carrier complexes too much, resulting in a flux decrease. Layers consisting of 100 wt \% PVC show, nevertheless, a nitrate flux. This might mean that the layer is permeable to nitrate and chloride, but it might also be an indication of a transfer of LM-phase from the support to the PVC, thereby plasticizing the PVC. This plasticization, or the uptake of LM-phase, might be a necessary condition for transport of nitrate by the carrier.

In Table $\mathrm{V}$, also the nitrate fluxes after 1 day of degradation are given. In all cases, these fluxes are very low. So, despite the presence of the PVC gel layer, carrier is lost from the liquid membrane into the aqueous phases. These physically cross-

Table V Nitrate Fluxes and Stability of SLMs with a PVC Gel Layer

\begin{tabular}{|c|c|c|c|c|}
\hline $\begin{array}{l}\text { PVC Content }{ }^{\mathrm{a}} \\
\quad(\text { wt } \%)\end{array}$ & PVC-Type & $\begin{array}{c}\text { Initial Flux } \\
\left(10^{-10} \mathrm{~mol} \mathrm{~cm}^{-2} \mathrm{~s}^{-1}\right)\end{array}$ & $\begin{array}{l}\text { Flux After } 1 \text { Day } \\
\left(10^{-10} \mathrm{~mol} \mathrm{~cm}^{-2} \mathrm{~s}^{-1}\right)\end{array}$ & $\begin{array}{l}\text { LM-Loss } \\
\quad(\mathrm{mg})\end{array}$ \\
\hline 0 & - & 15.9 & 1.3 & $19.7(7.0 \%)$ \\
\hline 0 & - & 15.1 & 1.0 & $18.2(6.3 \%)$ \\
\hline 40 & PVC-HMW & 10.7 & 1.3 & 21.3 \\
\hline 40 & PVC-HMW & 11.0 & 2.7 & 13.3 \\
\hline 40 & PVC-HMW & 11.2 & 2.3 & 11.3 \\
\hline 40 & PVC-carb & 10.8 & 2.5 & 11.6 \\
\hline 40 & PVC-carb & 10.8 & 2.3 & 10.1 \\
\hline 40 & PVC-carb & 10.1 & 0.5 & 10.3 \\
\hline 40 & PVC-carb & 8.7 & 0.7 & 8.7 \\
\hline 100 & PVC-carb & 9.4 & 0.7 & 3.4 increase \\
\hline 100 & PVC-carb & 9.5 & 0.7 & 5.5 increase \\
\hline
\end{tabular}

Support, Accurel; carrier, TOMA-Cl. Gelation by evaporation of THF. Casting knife thickness $100 \mu \mathrm{m}$. 
Table VI Nitrate Fluxes and Stability of SLMs with a Chemically Crosslinked PVC Gel Layer

\begin{tabular}{|c|c|c|c|c|c|c|}
\hline eq DCC & eq PDA & eq DDDA & $\begin{array}{l}\text { Casting Knife } \mathrm{e}^{\mathrm{a}} \\
(\mu \mathrm{m})\end{array}$ & Initial Flux & Flux After 1 Day & $\begin{array}{l}\text { LM-Loss } \\
\text { (mg) }\end{array}$ \\
\hline 0.82 & - & 0.82 & 100 & 14.2 & 0.8 & 14.0 \\
\hline 0.82 & - & - & 100 & 11.8 & 0.8 & 12.8 \\
\hline 0.82 & - & - & 100 & 11.7 & 0.6 & 13.2 \\
\hline 4.1 & 0.82 & - & 100 & 11.5 & 0.9 & 10.1 \\
\hline 4.1 & 0.82 & - & 100 & 10.2 & 0.5 & 15.8 \\
\hline 0.82 & - & - & 50 & 13.9 & 0.9 & 13.3 \\
\hline 0.82 & - & - & 50 & 14.1 & 1.0 & 13.4 \\
\hline 4.1 & 0.82 & - & 50 & 13.4 & 1.1 & 13.7 \\
\hline 4.1 & 0.82 & - & 50 & 13.1 & 0.8 & 12.9 \\
\hline
\end{tabular}

Support, Accurel, carrier, TOMA-Cl. Polymer concentration in gel layer, $40 \mathrm{wt} \%$. Gelation by evaporation of THF. Eq DCC, PDA, and DDDA, the amounts of activator and crosslinker in equivalents to the amount of carboxyl groups in the PVC. Fluxes in $10^{-10} \mathrm{~mol} \mathrm{~cm}^{-2} \mathrm{~s}^{-1}$.

${ }^{\text {a }}$ Slit height of the casting knife used.

linked PVC gel networks are not able to improve the stability since no flux could be detected any more after 1 day of degradation. This is opposite to the results of Neplenbroek et al. where, with these layers and TeOA as carrier, an improvement of stability could already be detected for PVC layers which were not chemically crosslinked. ${ }^{1,3}$ The absolute mass losses show a tendency to be somewhat lower than those of uncoated membranes. However, the increase in weight measured for layers of $100 \mathrm{wt} \% \mathrm{PVC}$ are not understood. Finally, it must be mentioned that the experiments with the different types of PVC show no differences in initial flux and SLM stability.

\section{Gel Layers with Chemical Crosslinking}

Since PVC gel layers without a chemical crosslinking were not effective in stabilizing the studied SLMs, we tried to shorten the mesh size of the gels by a chemical crosslinking. The amounts of crosslinker and activator were taken from Neplenbroek et al. ${ }^{1,7}$ Due to a change in the amount of carboxyl groups in our PVC compared to their PVC-carb, the amounts were somewhat lower. Nevertheless, a chemical crosslinking took place, indicated by the rapid visual increase in viscosity, and solidification after a while, of the PVC/LMphase mixture when DCC and amine were added.

Results concerning the chemically crosslinked membranes are shown in Tables VI and VII for two PVC concentrations investigated (40 wt \% and 50 wt \% PVC-carb, respectively). Much higher PVC concentrations were very difficult to achieve as a result of the high viscosity of the mixture and, consequently, difficulties in obtaining an uniformly cast gel layer. The thicknesses mentioned in Table VI and VII are the slit heights of the casting knives. After evaporation and crosslinking the thicknesses are most likely smaller.

Tables V-VII demonstrate the small decrease in initial flux as a result of the resistance of the gel layer. The thickness of the layer is still too high to obtain the same initial flux as for an uncoated SLM as observed by Neplenbroek et al. for gelled SLMs using TeOA-Br as carrier. ${ }^{1,7}$ When the crosslinking is carried out using DDDA as crosslinking agent, fluxes are slightly higher than for PDA, both for 40 and $50 \mathrm{wt} \%$ PVC. This might be due to the larger length of the crosslinking agent resulting in a somewhat "more open" structure. The use of a casting knife with a slit height of $50 \mu \mathrm{m}$ results in a small increase in initial flux compared to the knife with the $100 \mu \mathrm{m}$ slit. The lower the thickness of the gel layer, the lower the resistance and the higher the initial flux will be. SEM observations and thickness measurements with a micrometer confirmed the decrease in thickness.

It is clear from Tables VI and VII that a chemical crosslinking of the PVC does not result in any improvement of SLM stability. In all cases, the flux after one day is very low compared to the initial flux, and the absolute LM losses are almost the same as for uncoated SLMs (Table V). In case emulsion formation of the LM-phase is possible and it is the only or most important cause of the instability of TOMA-SLMs, gelation should have resulted in a stability improvement. This is not 
Table VII Nitrate Fluxes and Stability of SLMs with a Chemically Crosslinked PVC Gel Layer

\begin{tabular}{|c|c|c|c|c|c|c|}
\hline eq DCC & eq PDA & eq DDDA & $\begin{array}{c}\text { Casting Knife }{ }^{\mathrm{a}} \\
(\mu \mathrm{m})\end{array}$ & Flux Initial & Flux After 1 Day & $\begin{array}{l}\text { LM-Loss } \\
\quad(\mathrm{mg})\end{array}$ \\
\hline 4.1 & 0.82 & - & 100 & 10.2 & 0.9 & 19.7 \\
\hline 4.1 & 0.82 & - & 100 & 9.8 & 0.5 & 17.3 \\
\hline 0.82 & - & - & 100 & 12.2 & 0.8 & 19.1 \\
\hline 0.82 & - & - & 100 & 12.5 & 0.7 & 16.7 \\
\hline 0.82 & - & - & 100 & 12.4 & 0.6 & 11.8 \\
\hline 0.82 & - & - & 100 & 12.5 & 0.8 & 12.3 \\
\hline 0.82 & - & 0.82 & 100 & 12.8 & 0.8 & 11.7 \\
\hline 0.82 & - & 0.82 & 100 & 12.8 & 0.9 & n.d. \\
\hline 0.82 & - & 0.82 & 50 & 13.7 & 0.8 & 14.3 \\
\hline 0.82 & - & 0.82 & 50 & 14.3 & 0.9 & 13.8 \\
\hline
\end{tabular}

Support, Accurel; carrier, TOMA-Cl. Polymer concentration in gel layer, $50 \mathrm{wt} \%$. Gelation by evaporation of THF. Eq DCC, PDA, and DDDA to carboxyl groups. Fluxes in $10^{-10} \mathrm{~mol} \mathrm{~cm}^{-2} \mathrm{~s}^{-1}$.

a Slit height of the casting knife used; n.d., not determined.

observed. Apparently, either the emulsion formation hypothesis of Neplenbroek et al. ${ }^{1,3}$ is incorrect or there are other mechanisms responsible for the degradation of TOMA-SLMs.

When systems using TOMA-Cl as carrier are very unstable, it might be possible that the stabilizing effect of an interfacial gel layer is masked when in both cases (with and without layer) the flux after one day of degradation is very low. Therefore, a number of experiments were carried out without a degradation step in between the two flux measurements, i.e., after measuring the initial flux the feed solution is replaced by a fresh $4 \times 10^{-3} M \mathrm{NaNO}_{3}$ solution when the flux after one day had to be determined. The results are summarized in Table VIII. Despite the low flux after 1 day, the LM losses show a tendency to decrease with increasing PVC content in the gel layer. Due to the relatively large thickness of the gel layer, initial fluxes are lowered compared to uncoated SLMs. Comparing Tables V-VIII, fluxes after 1 day without degradation are slightly higher than with degradation. The absolute LM losses seem to decrease when no degradation step is carried out, although the differences are marginal for 0 and 40 wt \% PVC. The determined mass decrease is somewhat lower only in the 70 wt \% case. Nevertheless, here the flux after one day is almost zero, which is an indication for a large loss of carrier.

To check whether loss of carrier also takes place at the strip side of the membrane, a double layer membrane was prepared with two gel layers on each side of the SLM. Due to the gel layer preparation method, it is difficult to apply the second layer on one and the same membrane without damaging the first layer. Apart from this, the other side of the SLM is usually not flat, and this might cause problems in preparing a uniform gel layer. For these reasons, two SLMs with a single,

Table VIII Nitrate Fluxes and Stability of SLMs with a PVC Gel Layer

\begin{tabular}{ccccc}
\hline $\begin{array}{c}\text { PVC Content } \\
(\text { wt } \%)\end{array}$ & $\begin{array}{c}\text { DDC/PDA } \\
(\mathrm{eq})^{\mathrm{a}}\end{array}$ & $\begin{array}{c}\text { Flux Initial } \\
\left(10^{-10} \mathrm{~mol} \mathrm{~cm}^{-2} \mathrm{~s}^{-1}\right)\end{array}$ & $\begin{array}{c}\text { Flux After 1 Day } \\
\left(10^{-10} \mathrm{~mol} \mathrm{~cm}^{-2} \mathrm{~s}^{-1}\right)\end{array}$ & $\begin{array}{c}\text { LM-Loss } \\
(\mathrm{mg})\end{array}$ \\
\hline 0 & $0 / 0$ & 16.1 & 2.9 & $12.2(4.3 \%)$ \\
0 & $0 / 0$ & 16.1 & 3.1 & $13.9(4.9 \%)$ \\
40 & $4.1 / 0.82$ & 9.6 & 1.6 & 10.3 \\
40 & $4.1 / 0.82$ & 9.9 & 1.9 & 4.7 \\
70 & $4.1 / 0.82$ & 11.3 & 1.9 & 5.0 \\
70 & $4.1 / 0.82$ & 10.2 & 1.8 & 0.0 \\
\hline
\end{tabular}

Support, Accurel; carrier, TOMA-Cl. PVC, PVC-carboxylated. Gelation by evaporation of THF. Casting knife slit height, 100 $\mu \mathrm{m}$. No degradation step between the flux measurements.

${ }^{a}$ Equivalents to carboxyl groups in PVC. 
Table IX Results of Permeability Measurements of Two-Layered, Double SLMs

\begin{tabular}{cccc}
\hline Membrane & $\begin{array}{c}\text { Flux Initial } \\
\left(10^{-10} \mathrm{~mol} \mathrm{~cm}^{-2} \mathrm{~s}^{-1}\right)\end{array}$ & $\begin{array}{c}\text { Flux After 1 Day } \\
\left(10^{-10} \mathrm{~mol} \mathrm{~cm}^{-2} \mathrm{~s}^{-1}\right)\end{array}$ & $\begin{array}{c}\text { Flux After 4 Days } \\
\left(10^{-10} \mathrm{~mol} \mathrm{~cm}^{-2} \mathrm{~s}^{-1}\right)\end{array}$ \\
\hline 1 & 6.2 & 0.3 & 0.1 \\
2 & 6.1 & 0.5 & 0.2 \\
\hline
\end{tabular}

Support, Accurel. Carrier, TOMA-Cl; top layer, PVC-carb (40 wt \%); 4.1 eq. DCC, 0.82 eq. PDA. Gelation by evaporation of THF. Casting knife slit height, $100 \mu \mathrm{m}$.

chemically crosslinked gel layer were attached to one another, the layers facing the feed and strip sides. Results are given in Table IX.

Table IX demonstrates the reduction of the flux by a factor of two, when these data are compared to those in Table VI. Since the thickness of the double layer membrane is twice that of the usual one-layer SLM, only half of the flux was expected when the two membranes were well stuck together. Again the stability of these membranes is not increased. Therefore, these experiments do not give any definitive answer to the question whether carrier is lost as well to the strip phase.

\section{DISCUSSION}

In the introduction, it was postulated that the nitrate flux would be a function of the polymer concentration and the crosslink density. The results in Tables III-IV and Figure 3 for homogeneously gelled SLMs show a clear dependence on the polymer concentration: the higher the polymer concentration, the lower the initial nitrate flux. This is a result of an increase in the number of "obstacles" present in the gel, resulting in an increase in tortuosity of the membrane. The carrier complexes have to travel a longer pathway through the membrane and the flux will decrease. For SLMs with an interfacial, chemically crosslinked gel layer, the dependence of the initial flux on the polymer concentration is not clear, as is the dependence on the type and amount of chemical crosslinking agent. This indicates that the open structure of the gel network, despite the increase in chemical crosslinks or length of the chemical crosslink, is not lowering down the diffusion rate of the carrier complex through the membrane too much, as was also observed by Neplenbroek et al. ${ }^{1,7}$ Their initial fluxes for gelled membranes, compared to the membranes without a gel layer, were higher than in this work due to the smaller thickness of the gel layer in their work.
The previously presented results show that gelation of the LM-phase, either homogeneously in the pores of the support or as an interfacial gel layer on the feed side, is not able to improve the stability of SLMs used in this study when TOMA$\mathrm{Cl}$ is used as carrier. This result is opposite to measurements by Neplenbroek et al., who found very promising results using a very similar carrier, TeOA-Br. ${ }^{1,7}$ The only difference between these two carriers is the replacement of one octyl group from TeOA-Br by a methyl group. This results in a carrier which is much more surface active than TeOA-Cl, and the use of TOMA-Cl therefore results in SLMs that are much more unstable when no stabilizing precautions are undertaken. The stabilizing effect of gelation seems to be dependent on the LM-phase used: already fairly stable systems can be stabilized, as shown by Neplenbroek et al., but no effect is seen when the SLM is too unstable, e.g., with TOMA-Cl as carrier. Obviously, the mechanism for degradation of SLMs with these carriers is different. TeOASLMs might degrade mainly by an emulsion formation process, which can be suppressed by gelation. On the other hand, other mechanisms might be responsible for the instability of TOMA-SLMs, which are not minimized by gelation.

The gelation was carried out in a way very similar to that of Neplenbroek et al. However, repeating their experiments using $\mathrm{TeOA}-\mathrm{Br}$ as carrier did not result in identical promising results: the initial flux of the SLM with the gel layer was lower than that without gel layer, and no stability improvement was observed in our experiments. This might indicate that the idea of gelation itself works, but that the minor differences between our experiments and theirs causes the discrepancies. First, the chemicals used by Neplenbroek et al. and ours may differ, but in most experiments the same chemicals (PVC, activator, diamine, carrier ) and support material (Accurel) were used. Nevertheless, batch numbers might differ, and as a result there might be minor differences in their 
properties. Although the amount of carboxyl groups for our PVC-carb was somewhat higher than that used by Neplenbroek ( 1.8 wt \% and 1.4 wt \%, respectively) and the amounts of activator and crosslinker somewhat lower, we do not believe this can explain the instability of both TOMA and TeOA SLMs with an interfacial gel layer. Furthermore, the gel layers were applied by means of a casting knife and not by spreading the gel with a tissue on the support. The casting knife method is considered to give a more uniform, although larger, gel layer thickness than the tissue method. This explains why we observed a small initial flux decrease; however, this does not explain the absence of a stability improvement. Another difference is the setup used for the flux measurements. Although some instability is introduced by replacing feed and strip solutions in between the flux measurements; this is also the case in the measurements of Neplenbroek. Besides, in our setup, accumulators were incorporated, which damped the pulsations of the pump and minimized vibrations of the SLM. These accumulators were not present in their setup.

It might be assumed that chemicals and working method used are not responsible solely for the fact that no stability improvements are observed with the gelled SLMs using TOMA-Cl as carrier. The whole idea of gelation was based on the presumption that SLM instability in the system is caused mainly by an emulsion formation of the LM-phase. ${ }^{1,3,7,24}$ Preventing the LM-meniscus from deformation would lead to an increase in stability, since emulsion formation was suppressed, and thus a more stable SLM would be the result. No effect on the stability at all was seen for SLMs with TOMA-Cl as carrier when the LM-interface was gelled either homogeneously or by means of an interfacial gel layer. By this gelation the macroscopic viscosity of the LM-phase was increased, and thus the emulsion formation should be reduced. Since no effect on stability was observed, there might be additional factors causing loss of carrier and solvent from the liquid membrane. Such factors might be found on a molecular level, e.g., dissolution of carrier or membrane solvent in the aqueous phases. Such factors might explain why interfacially polymerized layers can improve the stability of TOMA-SLMs as presented before. ${ }^{30,31}$ These layers have a high degree of crosslinking as a result of the use of the trifunctional monomer trimesoylchloride $\mathrm{TMCl}$ as one of the monomers. This might result in layers with a very small "mesh-size" which can decrease the loss of LM-phase when caused by dissolution.

\section{CONCLUSIONS}

In contrast to the results of Neplenbroek et al., ${ }^{1,7}$ gelation of the LM-phase when TOMA-Cl is used as carrier is not effective in improving the stability of relatively unstable SLMs. By homogeneous gelation, the initial nitrate flux decreases as a result of the decrease in diffusion rate of the carrier complex with increasing polymer content. Due to the large thickness of the interfacial gel layers, the nitrate flux also decreased in this case. In both types of gelation, the flux after 1 day of degradation was almost zero. This might be an indication of other, additional causes of SLM instability when TOMA-Cl is used as carrier.

\section{REFERENCES}

1. A. M. Neplenbroek, Ph.D. dissertation, University of Twente, 1989.

2. A. M. Neplenbroek, D. Bargeman, and C. A. Smolders, Desalination, 79, 303 (1990).

3. A. M. Neplenbroek, D. Bargeman, and C. A. Smolders, J. Membr. Sci., 67, 133 (1992).

4. A. J. B. Kemperman, D. Bargeman, Th. van den Boomgaard, and H. Strathmann, Sep. Sci. Technol., 31, 2733 (1996).

5. F.-F. Zha, A. G. Fane, and C. J. D. Fell, J. Membr. Sci., 107, 75 (1995).

6. A. M. Neplenbroek, D. Bargeman, and C. A. Smolders, Chem. Mag., 570 (1989).

7. A. M. Neplenbroek, D. Bargeman, and C. A. Smolders, J. Membr. Sci., 67, 149 (1992).

8. T. Tanaka, Scientific American, January, 110 (1981).

9. R. Bloch, A. Finkelstein, O. Kedem, and D. Vofsi, Ind. Eng. Chem., Proc. Des. Dev., 6, 231 (1967).

10. D. Vofsi, O. Kedem, R. Bloch, and S. Marian, J. Inorg. Nucl. Chem., 31, 2631 (1969).

11. J. Jagur-Grodszinski, S. Marian, and D. Vofsi, Sep. Sci., 8, 33 (1973).

12. D. Vofsi and J. Jagur-Grodzinski, Naturwissenschaften, 61, 25 (1974).

13. M. Sugiura, J. Coll. Int. Sci., 81, 385 (1981).

14. M. Sugiura and T. Yamaguchi, J. Coll. Int. Sci., 96, 454 (1983).

15. M. Sugiura, M. Kikkawa, and S. Urita, Sep. Sci. Technol., 22, 2263 (1987).

16. M. Sugiura, M. Kikkawa, and S. Urita, J. Membr. Sci., 42, 47 (1989). 
17. H. Gankema, Ph.D. dissertation, University of Twente, 1995.

18. S. Sakohara, F. Muramoto, S. Sakai, M. Yoshida, and M. Asaeda, in Polymer Gels: Fundamentals and Biomedical Applications, D. DeRossi, K. Kajiwara, Y. Osada, and A. Yamauchi, Eds., Plenum Press, New York, 1991, p. 161.

19. S. Matson, E. Lee, D. Friesen, and D. Kelly, Acid Gas Scrubbing by Composite Solvent-swollen Membranes, US Patent 4,717,166, 12 April, 1988.

20. U. Oesch and W. Simon, Anal. Chem., 52, 692 (1980).

21. A. Walter, J. Polym. Sci., 13, 207 (1954).

22. U. Fiedler and J. Ruzicka, Anal. Chim. Acta, 67, 179 (1973).

23. H. J. Nielsen and E. H. Hansen, Anal. Chim. Acta, 85, 1 ( 1976 ).

24. L. Bromberg, G. Levin, and O. Kedem, J. Membr. Sci., 71, 41 (1992).
25. G. Levin and L. Bromberg, J. Appl. Polym. Sci., 48, 335 (1993).

26. A. J. B. Kemperman, Ph.D. dissertation, University of Twente, the Netherlands, 1995.

27. M. C. Wijers, Ph.D. dissertation, University of Twente, the Netherlands, 1996.

28. A. M. Neplenbroek, D. Bargeman, and C. A. Smolders, J. Membr. Sci., 67, 121 (1992).

29. W. Titow, in PVC Plastics. Properties, Processing and Applications, Elsevier, London, 1990, Chaps. 4 and 5.

30. A. J. B. Kemperman, Th. van den Boomgaard, and H. Strathmann, in Proceedings Euromembrane '95, W. R. Bowen, R. W. Field, and J. A. Howell, Eds., Centre for Continuing Education, University of Bath, UK, Vol. 1, 1995, p. 250.

31. A. J. B. Kemperman, H. H. M. Rolevink, D. Bargeman, Th. van den Boomgaard, and H. Strathmann, J. Membr. Sci., submitted. 